\title{
Parallelization of the Algorithm k-means Applied in Image Segmentation
}

\author{
Cristian José López Del Álamo \\ La Salle University \\ National University of San Agustin \\ Arequipa, Peru
}

\author{
Lizeth Joseline Fuentes Pérez \\ National University of San Agustin \\ Arequipa, Peru
}

\author{
Luciano Arnaldo Romero Calla \\ National University of San Agustin \\ Arequipa, Peru
}

\begin{abstract}
Algorithm k-means is useful for grouping operations; however, when is applied to large amounts of data, its computational cost is high. This research propose an optimization of $k$-means algorithm by using parallelization techniques and synchronization, which is applied to image segmentation. In the results obtained, the parallel $k$-means algorithm, improvement $50 \%$ to the algorithm sequential $k$-means.
\end{abstract}

\section{General Terms:}

speedup

\section{Keywords:}

parallelization, $k$-means, segmentation, images

\section{INTRODUCTION}

Several unsupervised learning algorithms have been proposed, which divide the set of objects in a number of groups according to an optimization criterion.

The grouping is defined, generally, as a process of organizing objects into groups whose members exhibit some kind of similarity [6]. The goal of clustering is to divide the set of objects, which have attributes associated to multidimensional vectors, into homogeneous groups so that patterns within each group are similars.

$K$-means is a algorithm widely used in clustering problems objects according to their attributes, in this regard, has been widely studied due to its applications in areas as machine learning [4], data mining [2], knowledge discovery [5], pattern recognition and classification [1], segmentation of medical images [3, 8], medical and general image [9].

Although, one biggest drawbacks of k-means algorithm is the high computational cost of calculating distances between all objects. For this reason, this research use parallelism and synchronization techniques in order to minimize the computational cost.
In the research, the parallel $k$-means algorithm, is applied to image segmentation, which seeks to group pixels with similar colors, improving the computational time in the segmentation, with large number of pixels, as well as, algorithm's iterations.

In the section 2 preliminary concepts are summarized, in the section 3 the methodology followed is presented and finally in the sections 4 and 5 the experiments and conclusions of the research is exposed.

\section{K-MEANS ALGORITHM AND SEGMETATION}

$K$-means is a partitional clustering algorithm, was proposed by Stuart Lloyd in 1957, although not published until 1982 [7]. A more efficient version was proposed and published in Fortran with Hartigan and Wong 1975 [4].

The k-means algorithm is an unsupervised clustering algorithm that classifies the input data, represented as multidimensional points, based on their inherent distance of each other [9].

The algorithm $k$-means has computational order equal to $O(n * k * s)$ where $n=$ number Iterations, $k=$ number Groups and $s=$ number of elements to group

The image segmentation algorithm is based on k-means, the pixels are clustered around $k$ centroids $C_{i}, \forall i=1 \ldots k$, which are obtained by running the algorithm 1 , where $N$ is the number of iterations, $K$ the number of centroids and $T$ the number of pixels.

The figure 1. show the image of Lena 1a segmented with two centroids $1 \mathrm{~b}$ with four centroids $1 \mathrm{c}$ and with eight centroids $1 \mathrm{~d}$

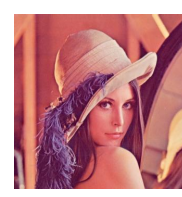

(a)

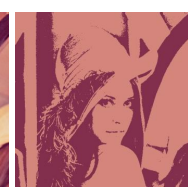

(b)

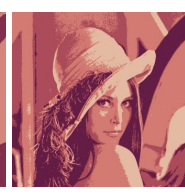

(c)

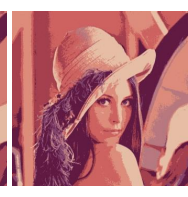

(d)
Fig. 1: K-means image segmentation 


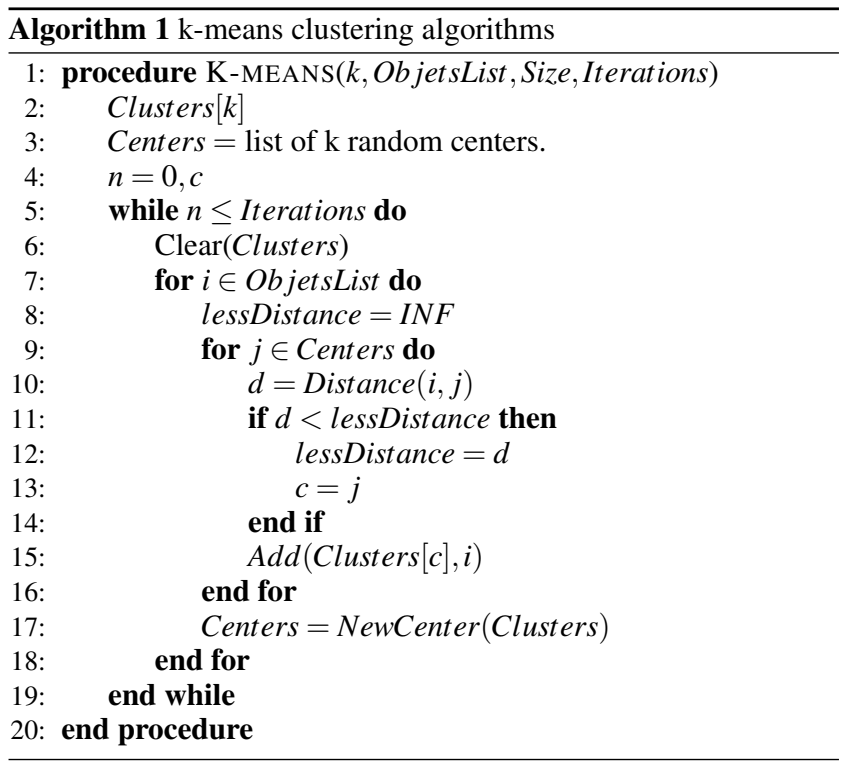

In the next section, the methodology used in this research will be explained, and in the sub section 3.2 the image segmentation algorithm using the sequential k-means and parallel k-means will be exposed.

\section{METHODOLOGY}

In this section the methodology used is described; all steps for the image segmentation with sequential and parallel $k$-means algorithm are showed in the figure 2

\subsection{Features vector of a pixel}

Given a set of test images, the RGB color space is used, and two types of features vector of each pixel is taken.

3.1.1 Features vector RGB. Lets $p_{i j}$ a image pixel, then $P_{i j}(r, g, v)$ is a vector, such that, $r, g$ and $b$ are the colors value in the pixel $(i, j)$ of the image and represent the descriptor from a color image. On the other hand, in the case of considerable color variation, represents a texture segmentation.

3.1.2 Features vector RGBXY. In this case, descriptor in $p_{i, j}$ is $P_{i, j}(r, g, b, x, y)$. This representation leads to a segmentation based on both, the color and position, of the pixel in the image.

\subsection{Segmentation}

The sequential and parallel $k$-means algorithm is applied in image segmentation process, considering the feature vectors RGBXY and RGB. With this two feature vectors, two different results are obtained, because RGBXY considers the position X,Y besides of the color, while RGB only consider the color of the pixels. Figure 3 show the segmenting of one image considering the feature vector RGB 3b and RGBXY 3a

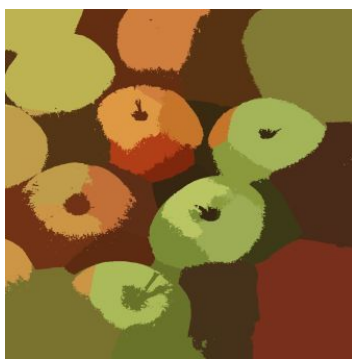

(a)

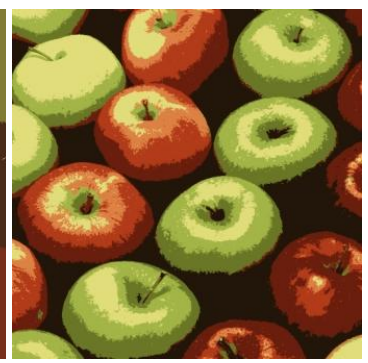

(b)
Fig. 3: Image Segmentation with $k$-means algorithm

\subsubsection{Secuential k-means. Described in the section 2}

3.2.2 Parallel k-means. The optimization process seek to parallelize the nearest centroid in line 7 of the algorithm 3 , because there are no dependencies between one iteration to another. On the other hand, the line 5 remains sequentially because each iteration depends on the centers that have been calculated in previous iterations. Furthermore, it may include calculating on-site of the new centers, if done by the average, at the same time, by calculating the belonging to each group. On the other hand, it is important to use a critical section on line 15 .

\subsection{Experiments and results}

After performing the respective tests with different images and number of threads, a result table and a graph showing the speedup of parallel algorithm is obtained. This process is explained in the section 4

\subsection{Comparison}

Analysis of the final results, conclusions and discussion, will be discussed in section 5

Figure 4 illustrates the input image $4 \mathrm{a} .1600 \times 1000$ pixels, which is applied to the parallel algorithm with 4 threads, 20 iterations and 2,4 and 8 centroids; segmenting into 2 groups $4 \mathrm{~b}$, groups $4 \mathrm{c}$ and 8 groups $4 \mathrm{~d}$ respectively.

\section{EXPERIMENT AND RESULT}

The tests were performed on an Intel (R) Core (TM) i3 2.27 $\mathrm{GHz}$ processor with $2.8 \mathrm{GiB}$ RAM. For the parallelization of the $k$-means algorithm was used the OpenMP library for $C++$ and images for testing as shown below.

In Figures 5 and 6 the application of image segmentation is shown with the parallel $K$-means algorithm. $5 \mathrm{a}$ and $6 \mathrm{a}$ are the original images, in $5 \mathrm{~b}$ and $6 \mathrm{~b}$ images are segmented with two centroids, in $5 \mathrm{c}$ and $6 \mathrm{c}$ with 4 centroids. Finally, in 5d with 32 centroids (some centroids may not have grouped pixels) and $6 \mathrm{~d}$ with 8 centroids.

After empirical testing images, is desired to obtain the optimal speedup for our algorithm, which is represented by the largest value of the inverse of the time to take a test run with $N$ threads. 

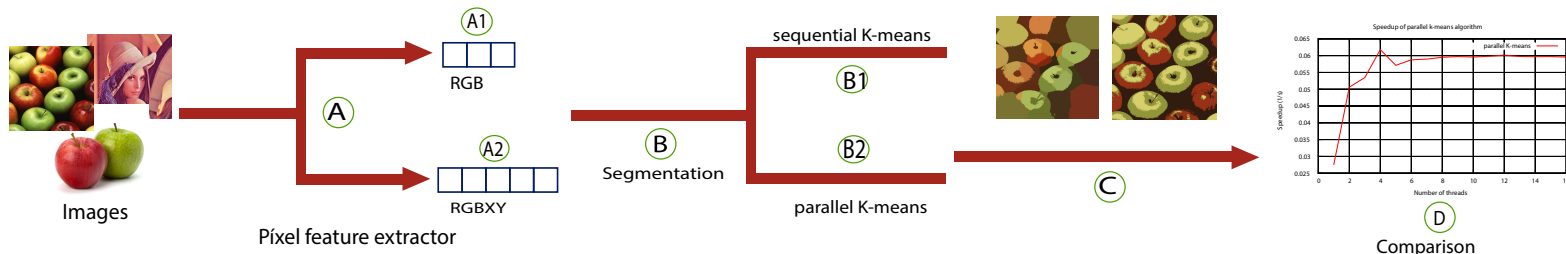

Fig. 2: Research methodology

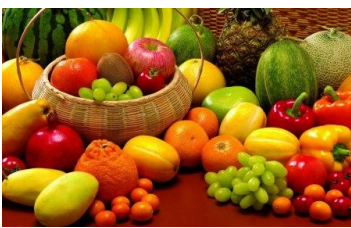

(a)

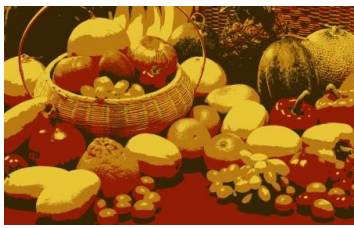

(c)

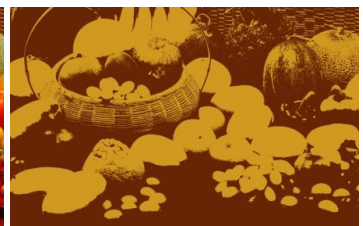

(b)

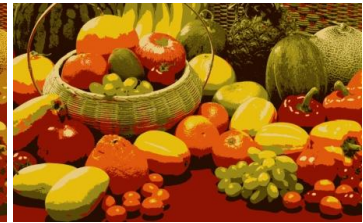

(d)

Fig. 4: image segmentation with parallel $k$-means algorithm

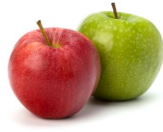

(a)

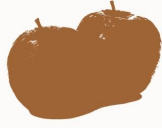

(b)

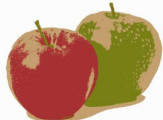

(c)

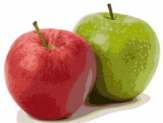

(d)
Fig. 5: Image segmentation with the parallel $k$-means algorithmo

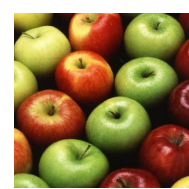

(a)

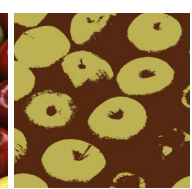

(b)

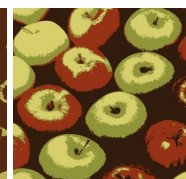

(c)

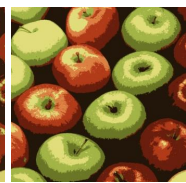

(d)
Fig. 6: Image segmentation with the parallel $k$-means algorithm

For it, will take of the Figure 7 the image $7 \mathrm{a}$ as test image, which has a dimension $1600 \times 1000$ pixels, the number of centroids will be 20 and the number of iterations 50 .

The segmentation process with different numbers of threads will be run, which vary from 1 to 16 . For each set of threads, 10 experiments were conducted and the result is taken as the average time for each of the experiments.

Figure $7 b$ illustrated the segmentation result for one of the tests. The test had a total time of $48.8454 \mathrm{~min}$.

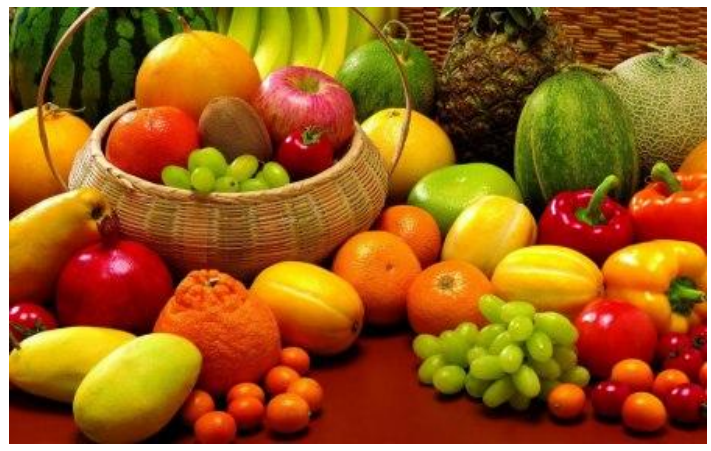

(a)

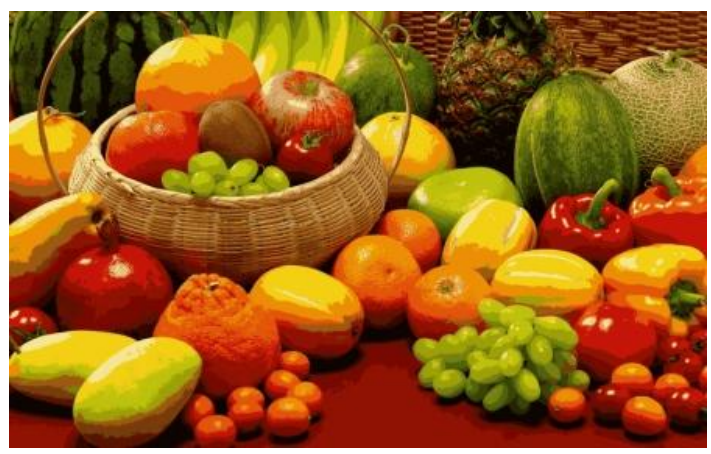

(b)

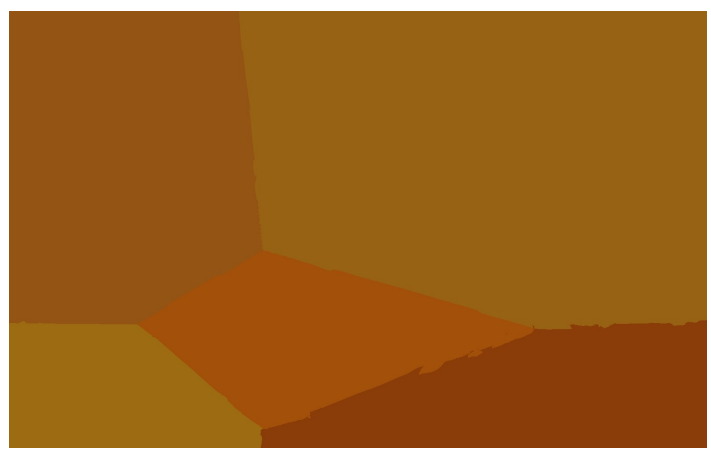

(c)

Fig. 7: Image segmentation with the parallel $k$-means algorithm

Table 1 show the result of the test, where you can see the average speedup for each number of threads, average time and improving respectively, compared to the sequential execution. The optimal speedup is in the test run with 4 threads (*), having improved $55 \%$ relative to the sequential algorithm and an average run- 
time $16.1881 s$. In (*) additional evidence that clearly show that the speedup decreases when the number of threads increases.

\begin{tabular}{rccc}
\hline Nro de hilos & Speedup & Time (s) & Improvement (\%) \\
\hline 1 & 0.0276079 & 36.2215 & 0.0000 \\
2 & 0.0506200 & 19.7550 & 45.4606 \\
3 & 0.0534600 & 18.7056 & 48.3577 \\
$(*) \mathbf{4}$ & $\mathbf{0 . 0 6 1 7 7 3 6}$ & $\mathbf{1 6 . 1 8 8 1}$ & $\mathbf{5 5 . 3 0 8 0}$ \\
5 & 0.0571196 & 17.5071 & 51.6666 \\
6 & 0.0587511 & 17.0209 & 53.0088 \\
7 & 0.0589585 & 16.9611 & 53.1739 \\
8 & 0.0595100 & 16.8039 & 53.6079 \\
9 & 0.0597033 & 16.7495 & 53.7581 \\
10 & 0.0595853 & 16.7827 & 53.6665 \\
11 & 0.0597975 & 16.7231 & 53.8310 \\
12 & 0.0601453 & 16.6264 & 54.0980 \\
13 & 0.0597482 & 16.7369 & 53.7929 \\
14 & 0.0596862 & 16.7543 & 53.7449 \\
15 & 0.0597196 & 16.7449 & 53.7708 \\
16 & 0.0595534 & 16.7916 & 53.6419 \\
$\mathbf{1 0 0}(*)$ & $\mathbf{0 . 0 5 3 7 7 5 0}$ & $\mathbf{1 8 . 5 9 6 0}$ & $\mathbf{4 8 . 6 6 0 3}$ \\
$\mathbf{1 0 0 0}(*)$ & $\mathbf{0 . 0 5 2 5 4 9 7}$ & $\mathbf{1 9 . 0 2 9 6}$ & $\mathbf{4 7 . 4 6 3 2}$ \\
$\mathbf{1 0 0 0 0}(*)$ & $\mathbf{0 . 0 3 7 4 0 8 1}$ & $\mathbf{2 6 . 7 3 2 2}$ & $\mathbf{2 6 . 1 9 8 0}$ \\
\hline
\end{tabular}

Table 1. : Tests results, (*) additional tests

Figure 8 shows the pictures of average speedup depending on the number of threads, and in the tablet 1 a clear improvement of the parallel algorithm is observed in relation to sequential algorithm.

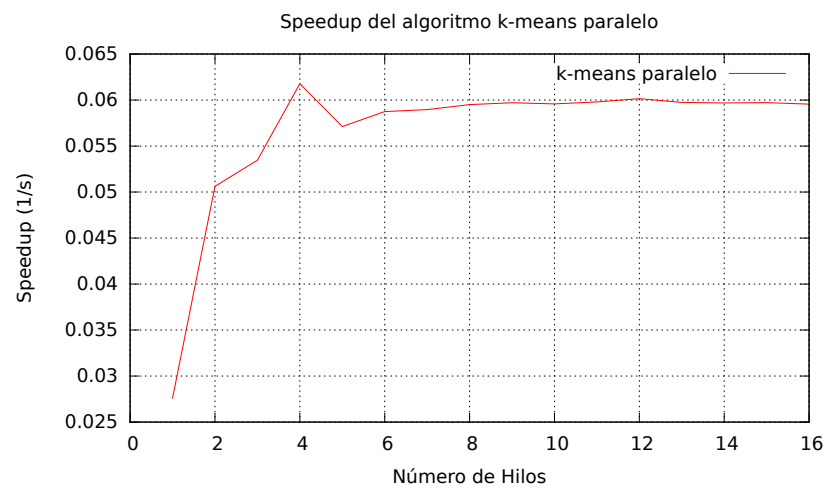

Fig. 8: Speedup of parallel k-means algorithm in image segmentation

\section{CONCLUSIONS}

From the experiments, is concluded that the parallel $k$-means algorithm with 4 threads, outperforms by $55 \%$ to the sequential $k$-means algorithm. In the test performed, in general, the the parallel $k$-means optimizes sequential $k$-means. In fact, for a small number of iterations, or small groups images is convenient to use the sequential algorithm. However, for large images where the processing of the segmentation and clustering is heavy is preferable to use parallel techniques.
From Table 1 is concluded that the optimal speedup is obtained with 4 threads, and to increase more threads, the performance decrease. Furthermore, is found that the parallel algorithm has better overall performance than the sequential. But also, the performance tends to decrease with the number of threads as shown in the tests.

\section{FUTURE WORK}

In future work, the parallel $k$-means algorithm will be applied on specific problems, such as, segmentation of satellite images. In that sense, will be developed techniques for distributed processing, in order to split an image of several mega bytes of size, on multiple machines, so that each run a part of the process of segmentation and clustering.

\section{Acknowledgment}

We thank Professor Wilber Ramos Lovón, Principal of School of Computer Science from National University of San Agustín, for his unconditional surrender to their students, to the National University of San Agustín and La Salle University for supporting this research.

\section{REFERENCES}

[1] Richard O. Duda and Peter E. Hart. Pattern Classification and Scene Analysis. John Wiley \& Sons Inc, 1 edition, February 1973.

[2] Usama M. Fayyad, Gregory Piatetsky-Shapiro, Padhraic Smyth, and Ramasamy Uthurusamy. Advances in Knowledge Discovery and Data Mining. The MIT Press, February 1996.

[3] A. Ferreira, J. M R S Tavares, and F. Gentil. A review of segmentation algorithms for ear image data. In Information Systems and Technologies (CISTI), 2012 7th Iberian Conference on, pages 1-6, 2012.

[4] J. A. Hartigan and M. A. Wong. Algorithm AS 136: A k-means clustering algorithm. Applied Statistics, 28(1):100-108, 1979.

[5] Mehmed Kantardzic. Data Mining: Concepts, Models, Methods and Algorithms. John Wiley \& Sons, Inc., New York, NY, USA, 2002.

[6] Aristidis Likas, Nikos Vlassis, and Jakob J. Verbeek. The global k-means clustering algorithm, 2001.

[7] S. Lloyd. Least squares quantization in pcm. IEEE Trans. Inf. Theor, 28(2):129-137, September 2006.

[8] H. P. Ng, S. H. Ong, K. W C Foong, P. S. Goh, and W.L. Nowinski. Medical image segmentation using k-means clustering and improved watershed algorithm. In Image Analysis and Interpretation, 2006 IEEE Southwest Symposium on, pages 6165, 2006.

[9] Suman Tatiraju and Avi Mehta. Image segmentation using kmeans clustering, em and normalized cuts. 